\title{
DIRECT NEWTON FINITE-ELEMENT/BOUNDARY-ELEMENT TECHNIQUE FOR MICRO-ELECTRO-MECHANICAL-ANALYSIS
}

\author{
N. R. Aluru and J. White \\ Department of Electrical Engineering and Computer Science \\ Massachusetts Institute of Tochnology, Cambridge, MA 02139
}

\begin{abstract}
In this paper we describe a new approach to self-consistent electromechanical simulation. The approach employs a finite-element method for mechanical analysis and a precorrected fast-Fourier transform accelerated boundary-element method for electrostatic analysis. The convergence of the new approach is compared with that of relaxation and surface-Newton methods and is shown to exhibit superior convergence behavior.
\end{abstract}

\section{INTRODUCTION}

In order to investigate design altematives, designers of novel MEMS structures need efficient, robust and easily used computer simulation tools. And since most of the structures of interest are geometrically complicated, electromechanically coupled, and are inherently three-dimensional, Micro-Electro-Mechanical CAD (MEMCAD) tool developers have been focussed on improving the usability, efficiency and robustness of coupled 3-D electromechanical analysis. In particular, finite-element based elastostatic analysis and accelerated boundary-element based electrostatic analysis have been combined using algorithms based on relaxation and a form of surface-Newton method [1], [2], [3]. Although the relaxation algorithm is easy to program, only requiring that data be passed back and forth between "black-box" elastostatic (e.g. ABAQUS [4]) and electrostatic (e.g. FASTCAP [5]) analysis programs, the algorithm diverges if the structure is too flexible or the electric fields are too large. Matrix-free surface-Newton methods preserve the "blackbox" nature of the relaxation algorithm and have better convergence properties, but also have perturbation parameters which must be tuned for good performance [3]. In this paper, we present a directNewton based approach which is more robust and exhibits superior convergence behavior compared to relaxation and surface-Newton methods.

This paper is organized as follows: In the next section a brief overview is presented on the existing approaches to self-consistent electromechanical simulation. In Section III, the direct-Newton technique is described and numerical results are presented in Section IV. Finally, conclusions are presented in Section V.

\section{PREVIOUS APPROACHES}

\section{A. Relaxation Technique}

Relaxation is the simplest approach to electromechanical simulation as the coupled physical domains are analyzed separately. The surface pressures, which depend on the electrostatic charge, are assumed to be known when computing the structural deformation, and the structural deformations are assumed to be known when computing the electrostatic charge. Figure 1 summarizes the relaxation algorithm for electromechanical analysis. An advantage of the relaxation algorithm is that since the domains are analyzed separately, the numerical schemes can be different. For example, a very

This research was supported by ARPA under ONR contract DABT63-94C-0053 and FBI contract J-FBI-92-196, by SRC under contract SJ-558, and by grants from IBM and Digital Equipment Corporation.

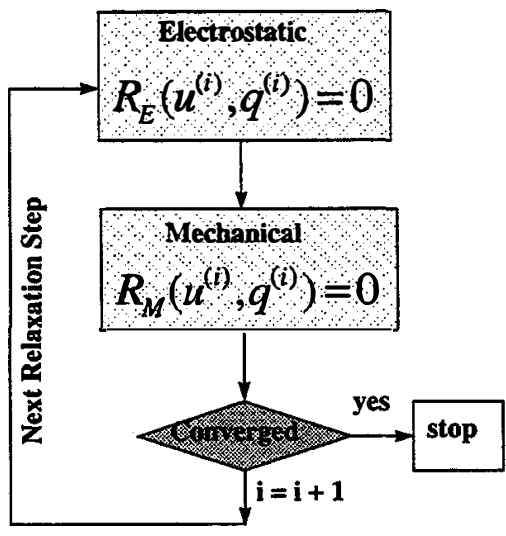

Figure 1. Relaxation algorithm for self-consistent electromechanical analysis. $R_{M}(u, q)$ and $R_{E}(u, q)$ denote the elastostatic(mechanical) and electrostatic equations respectively, $u$ is the deformation of the structure, and $q$ is the surface charge that produces the electrostatic force.

efficient relaxation scheme combines Galerkin finite-element elastostatic analysis with multipole/precorrected fast Fourier ransform acceleration technique for the electrostatic analysis [1], [2]. The availability of commercial simulators for each physical domain also makes relaxation algorithms appealing as they can be implemented very quickly using a black-box approach. The relaxation algorithm, however, is not robust because it converges very slowly or fails to converge in tightly coupled cases.

\section{B. Surface-Newton Technique}

The surface-Newton technique was designed to preserve the black-box nature of the relaxation algorithm but to improve its convergence [3]. The approach presented in [6] is similar to the surfaceNewton technique. The key idea in the surface-Newton approach is to reduce the dimensionality of the coupled problem from 3-D to 2-D and to employ a Newton technique to determine the surface node positions. Once the node positions on the structure surface are known, both the surface pressure and the interior node positions can be determined by decoupled electrostatic and elastostatic analysis. Therefore, we can write a surface-Newton iteration equation as

$$
R_{s}\left(u_{s}{ }^{k}\right)+\frac{\partial R_{s}}{\partial u_{s}}\left(u_{s}{ }^{k+1}-u_{s}{ }^{k}\right)=0
$$

where $u_{s}$ is the vector of surface displacements, $k$ is the iteration index, and

$$
R_{s}\left(u_{s}{ }^{k}\right)=u_{s}{ }^{k}-S u r f\left[R_{M}\left(u_{o s}, R_{E}\left(u_{s}{ }^{k}+u_{o s}, V\right)\right] .\right.
$$

Here, $u_{o s}$ is used to denote the surface of the initial structure and the function $S u r f$ extracts $u_{s}$ from $u$.

A matrix-free Generalized Conjugate Residual (GCR) iterative method [5] can then be used to solve (2), in which case each GCR 
iteration involves forming a matrix-vector product. The matrixvector product can be computed using finite-differences i.e.

$$
\frac{\partial R}{\partial u} * r \approx \frac{1}{\theta}(R(u+\theta * r)-R(u))
$$

where

$$
\begin{aligned}
& \theta=\operatorname{sign}(u * r) * \min \left(1, \frac{a\|u\|}{\|r\|}, \frac{b\|R(u)\|}{\|r\|}\right) \\
& a \in(0.01,0.5) \quad b \in(0.1,1)
\end{aligned}
$$

The surface-Newton technique suffers from several drawbacks. First, the algorithm's robustness is very sensitive to the precise choice of $\theta$. If $\theta$ is too large, the nonlinearities in $R$ will compt the derivative estimate. If $\theta$ is too small, the small numerical errors in solving the electrostatic and elastostatic problems will compt the derivative estimate. Second, each surface Newton iteration requires a complete solution of the nonlinear elastostatic and electrostatic equations. Thus the approach may not be very efficient. Third, the Jacobian employed in the surface-Newton technique is not exactly identical to the Jacobian of the fully-coupled method presented in the next section. Hence, the convergence of the surface-Newton technique may not be optimal for all cases.

\section{NEW APPROACH: DIRECT-NEWTON TECHNIQUE}

The key idea in the direct-Newton or coupled approach is to account for all the coupling terms between electrical and mechanical systems and to compute the coupling efficiently and exactly. Figure 2 summarizes the coupled algorithm for electromechanical analysis. A coupled approach has been attempted before [7] by employing a finite element method for both the electrostatic and elastostatic equations. This approach may not be computationally very efficient as a finite-element method, in comparison to a multipole-accelerated boundary-element method, would require the construction of an exterior mesh for the electrostatic analysis. A computationally efficient approach of a hybrid finite-element/boundary-element method is employed in the direct-Newton technique.

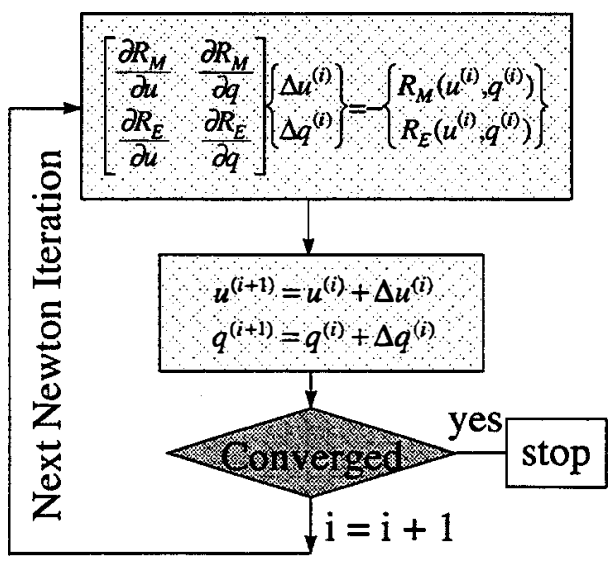

Figure 2. A coupled algorithm for self-consistent electromechanical analysis.

As shown in Figure 2, the outer Newton iteration solves the nonlinear coupled system, and the linear system within each Newton iteration is solved by employing a generalized-minimal-residual (GMRES) algorithm [8]. The coupled system Jacobian can be divided into four parts: the entirely elastostatic part, often referred to as the stiffness or deformation-coefficient matrix, which determines the change in force due to geometric perturbations; the entirely electrostatic part, which determines the change in potential due to perturbations in surface charge; the mechanical to electrical part, which determines the change in force due to perturbation in surface charge; and the electrical to mechanical part, which determines the change in potentials due to geometric perturbations. The deformation-coefficient matrix is computed by employing a Galerkin finite-element formulation. The formulation accounts for both material and geometric nonlinearities and is summarized in Figure 3(a). A sparse storage scheme [9] is employed to store the deformationcoefficient matrix. The mechanical to electrical coupling, $\frac{\partial R_{M}}{\partial q}$, is computed by calculating the variation of the elastostatic residual equation with respect to the charge and integrating over the boundary elements on the mechanical domain.

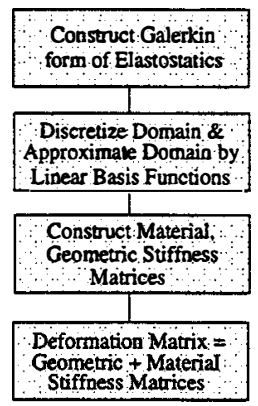

(a)

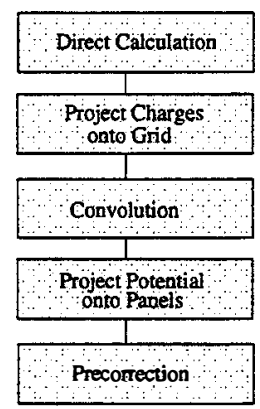

(b)
Figure 3. (a) Computation of the deformation coefficient matrix (b) Computation of the matrix-vector product employing precorrectedFFT technique.

The electrostatic or potential coefficient matrix is not needed explicitly as the GMRES algorithm, within each Newton iteration, requires only a matrix-vector product involving the matrix, $\frac{\partial R_{E}}{\partial q}$, and the charge increment vector, $\Delta q$. In earlier approaches to eleciromechanical simulations [1], [2], [3], a multipole algorithm is employed to efficiently compute the matrix-vector product. In [10], a precorrected Fast Fourier Transform (FFT) algorithm for computing the matrix-vector product was presented, and as compared to multipole algorithm, has been shown to be faster and utilizes less memory. The precorrected-FFT algorithm is employed in our direct-Newton technique and the computation of the matrix-vector product with this approach is summarized in Figure 3(b).

The electrical to mechanical coupling term can be computed by employing a matrix-free approach. The residual equation for the electrostatic system is given as

$$
R_{E}=P q-\bar{p}=0
$$

where $P$ is the potential-coefficient matrix, $q$ is the charge vector and $\bar{p}$ is the vector of applied potentials. An approach to compute the electrical to mechanical coupling term is given as

$$
\frac{\partial R_{E}}{\partial u} \cdot \Delta u^{(i)} \approx \frac{P\left(u+\epsilon \Delta u^{(i)}\right) q-P(u) q}{\epsilon}
$$

where $\epsilon$ is a small parameter and plays a similar role as $\theta$ does in the surface-Newton technique. However, we will show in the results section that the choice of $\epsilon$ is not critical for the robusmess of the method. In order to compute the marrix-vector product, $\frac{\partial R_{E}}{\partial u} \cdot \Delta u^{(}$, 
accurately a small value of $\epsilon$ is desired and is determined through an optimization problem [11]. For well-scaled residuals, an optimal value of $\epsilon$ is $O\left(\epsilon_{m}^{1 / 2}\right)$, where $\epsilon_{m}$ is the machine precision.

According to Equation (5), the electrical to mechanical coupling can be computed by performing two matrix-vector products. The first matrix-vector product, $P(u) q$, is straight forward. The second marix-vector product, $P\left(u+\epsilon \Delta u^{(i)}\right) q$, can be obtained by perturbing the panel/conductorgeometry from $u$ to $u+\epsilon \Delta u^{(i)}$ and applying the precorrected-FFT algorithm.

\section{RESULTS}

The direct-Newton technique is compared with the relaxation technique for two examples: a beam over a ground plane structure and two silicon bars with one end of one silicon bar and the other silicon bar held fixed. To make the comparison accurate, a new relaxation code is developed that also employs a precorrected-FFT accelerated boundary-element method for the electrostatic equations, and the finite-element formulation for the elastostatic equations is similar in both the direct-Newton/coupled and relaxation codes.

Figure 4 compares the peak deflection obtained from the relaxation and coupledNewtonalgorithms for a $500 \mu \mathrm{m} \times 50 \mu \mathrm{m} \times 14.35$ $\mu \mathrm{m}$ beam positioned $1 \mu \mathrm{m}$ above a ground plane. The results are identical verifying the accuracy of the coupled solver. The deflection of the beam for an applied bias of $17.23 \mathrm{~V}$ is shown in Figure 5.

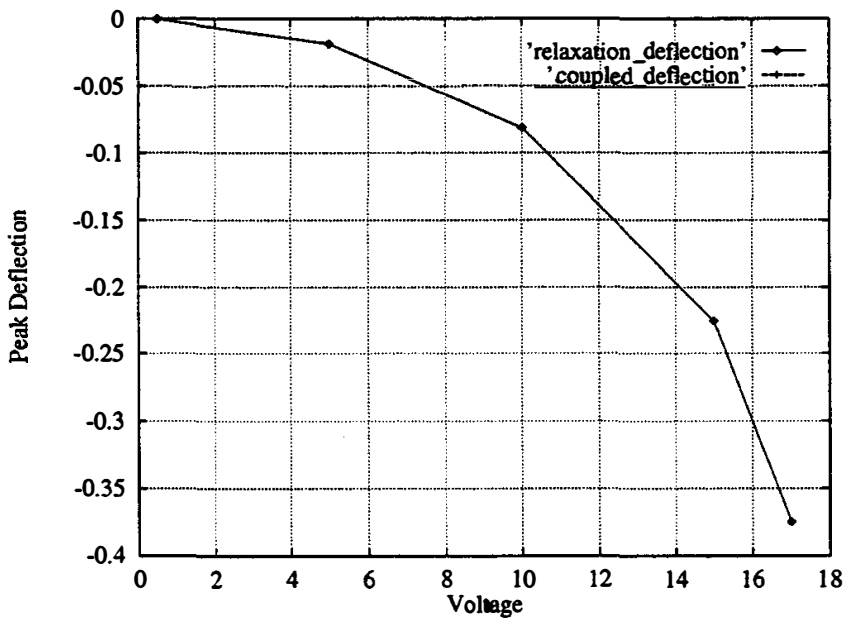

Figure 4. Comparison of peak deflections from relaxation and coupled algorithms for a beam over a ground plane structure. Note that the two curves overlap.

Figures 6 and 7 compare the convergence of the relaxation and

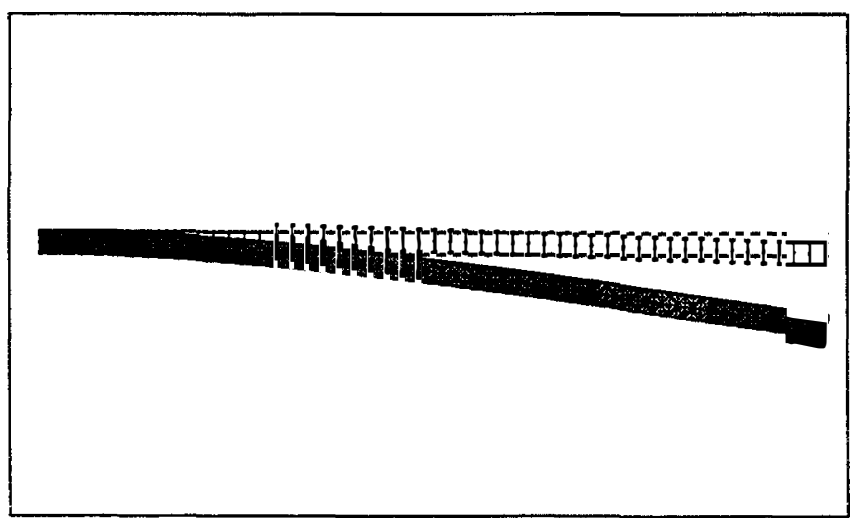

Figure 5. Deflecion of the beam for an applied bias of $17.23 \mathrm{~V}$. coupled algorithms for the beam and ground plane example. The pull-in for the structure is $17.24 \mathrm{~V}$ and note that closer to pull-in the relaxation algorithm converges slowly, but the coupled algorithm converges rapidly. The slow convergence of the relaxation algorithm, near pull-in, is due to the increased coupling between elastostatic and electrostatic systems. As the direct-Newton technique accurately accounts for all the coupling it exhibits rapid convergence behavior.

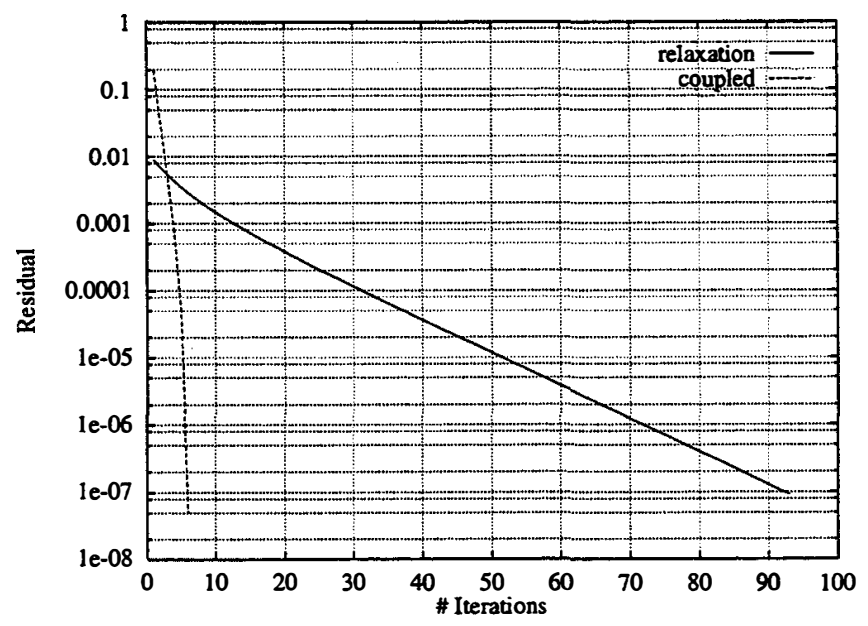

Figure 6. Convergence of relaxation and coupled algorithms for a beam and ground plane structure at $17.20 \mathrm{~V}$.

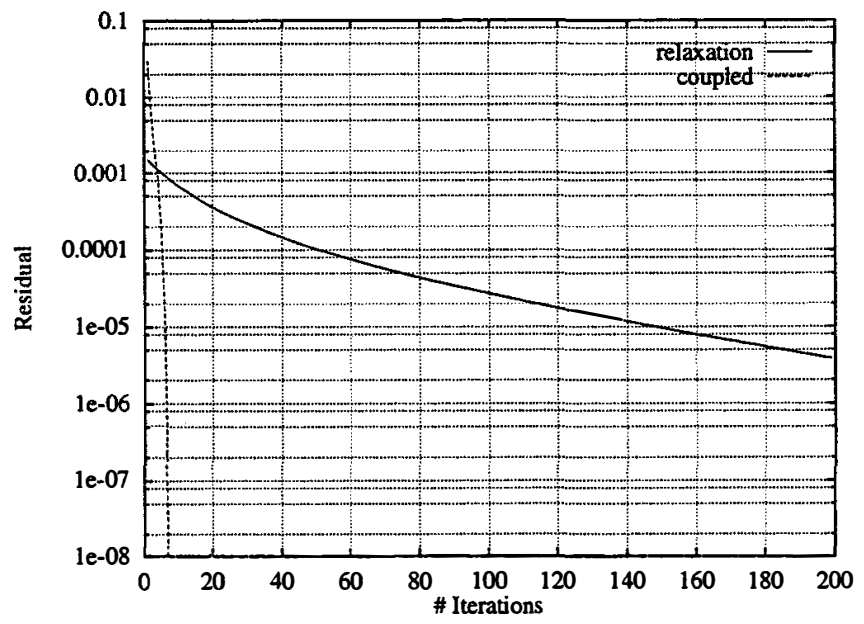

Figure 7. Convergence of relaxation and coupled algorithms for a beam and ground plane structure at $17.23 \mathrm{~V}$.

The direct-Newton technique employs a matrix-free approach to compute the electrical to mechanical computing. The matrix-free parameter, $\epsilon$, is a concern as the method may not work for all values of $\epsilon$. Figure 8 compares the convergence of the coupled algorithm for three different values of $\epsilon$. A value of $\epsilon=1.0$ requires only one more iteration for convergence as compared to using an optimal $\epsilon$. No noticeable convergence rate differences are observed between employing an optimal $\epsilon$ and an $\epsilon$ larger than optimal by a factor of 100. Hence, the choice of $\epsilon$ is not critical for the robustness of the method, unlike the surface-Newton technique.

Figure 9 compares the convergence of the relaxation and coupled algorithms for two silicon bars with a potential difference of $850 \mathrm{~V}$. The relaxation algorithm fails to converge for this example because of the strong coupling between mechanical and electrical domains at 


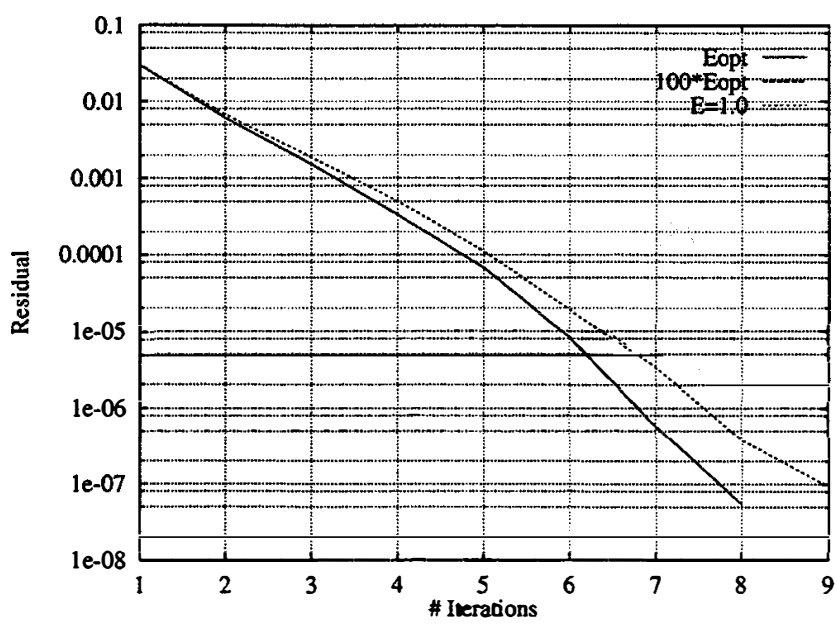

Figure 8. Comparison of convergence for different values of $\epsilon$. Eopt $=\operatorname{sqrt}\left(\epsilon_{m}\right), 100^{*} \mathrm{Eopt}=100 \cdot \operatorname{sqrt}\left(\epsilon_{m}\right)$, and $\mathrm{E}=1$ corresponds to $\epsilon=1.0$.

$850 \mathrm{~V}$. The coupled algorithm not only converges but converges very rapidly.

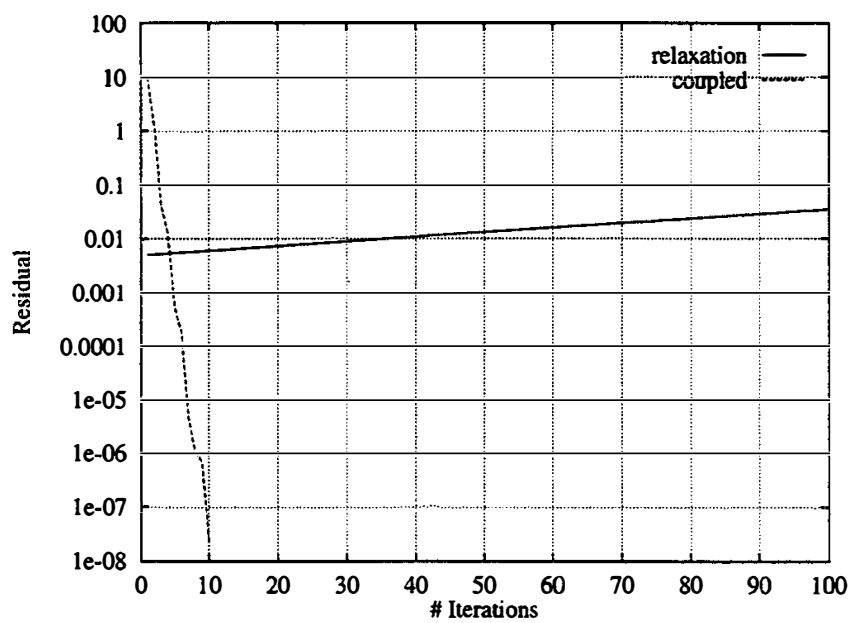

Figure 9. Convergence of relaxation and coupled algorithms for two silicon bars with a potential difference of $850 \mathrm{~V}$.

\section{CONCLUSION AND ACKNOWLEDGEMENTS}

The direct-Newton approach is shown to exhibit superior convergence behavior as compared to relaxation method and does not have the parameter sensitivity of the surface-Newton method. In addition, unlike the surface-Newton approach, the direct-Newton method does not require solving nonlinear elastostatic and a linear electrostatic problem in each Newton iteration. The approach presented in this paper to compute the electrical to mechanical coupling term may not be very efficient. We are presently exploring other approaches and they will be reported in a future publication. The efficiency of the direct-Newton technique can be further improved by employing acceleration techniques, such as preconditioners, and this is also being studied.

The authors would like to thank Dr. John R. Gilbert, Dr. Peter M. Osterberg, Joel R. Phillips and Professor Steven D. Senturia for many valuable discussions.

\section{REFERENCES}

[1] X. Cai, H. Yie, P. Osterberg, J. Gilbert, S. Senturia and J. White, "A relaxation/multipole-accelerated scheme for self-consistent electromechanical analysis of complex 3-D microelectromechanical structures," Proc. ICCAD 1993.

[2] J. R. Gilbert, R. Legtenberg and S. D. Senturia, "3D coupled electromechanics for MEMS: Applications of CoSolve-EM," Proc. MEMS 1995.

[3] H. Yie, X. Cai and J. White, “Convergence properties of relaxation versus the surface-Newton generalized-conjugate residual algorithm for self-consistent electromechanical analysis of 3-D micro-electro-mechanical structures" Proc. NUPAD V 1994.

[4] Hibbit, Karlsson and Sorenson, Inc., Providence, R.I.

[5] K. Nabors and J. White, "FastCap: A multipole-accelerated 3-D capacitance extraction program," IEEE Trans. CAD, vol. 10, pp. 1447-1459, 1991

[6] M. Bachtold, J. G. Korvink, J. Funk and H. Baltes, "New convergence scheme for self-consistent electromechanical analysis of iMEMS," Proc. IEDM 1995.

[7] H. U. Schwarzenbach, J. G. Korvink, M. Roos, G. Sartoris, and E. Anderheggen, "A microelectromechanical CAD extension for SESES," JMEMS, vol. 3, pp. 162-171, 1994.

[8] Y. Saad and M. H. Schultz, "GMRES: A generalized minimal residual algorithm for solving nonsymmetric linear systems," SIAM J. Sci. Stat. Comput., vol. 7, pp. 856-869, 1986.

[9] K. S. Kundert and A. Vincentelli, "Sparse User's Guide: A sparse linear equation solver," Univ. of California, Berkeley, 1988.

[10] J. R. Phillips and J. White, "A Precorrected-FFT method for capacitance extraction of complicated 3-D structures," Proc. ICCAD 1994.

[11] P. E. Gill, W. Murray and M. H. Wright, Practical Optimization, Academic Press, London, 1981. 